\title{
Fetal liver mesenchymal stem cells restore ovarian function in premature ovarian insufficiency by targeting MT1
}

\author{
Boxian Huang ${ }^{1,2^{*}+} \mathbb{D}, C$ Chunfeng Qian ${ }^{1 \dagger}$, Chenyue Ding $^{1 \dagger}$, Qingxia Meng ${ }^{1}$, Qinyan Zou ${ }^{1}$ and Hong Li $^{1 *}$
}

\begin{abstract}
Background: With the development of regenerative medicine and tissue engineering technology, almost all stem cell therapy is efficacious for the treatment of premature ovarian failure (POF) or premature ovarian insufficiency (POI) animal models, whereas little stem cell therapy has been practiced in clinical settings. The underlying molecular mechanism and safety of stem cell treatment in POI are not fully understood. In this study, we explored whether fetal mesenchymal stem cells (fMSCs) from the liver restore ovarian function and whether melatonin membrane receptor 1 (MT1) acts as a regulator for treating POI disease.

Methods: We designed an in vivo model (chemotherapy-induced ovary damage) and an in vitro model (human ovarian granulosa cells ( $(\mathrm{GGCS})$ ) to understand the efficacy and molecular cues of fMSC treatment of POI. Follicle development was observed by H\&E staining. The concentration of sex hormones in serum (E2, AMH, and FSH) and the concentration of oxidative and antioxidative metabolites and the enzymes MDA, SOD, CAT, LDH, GR, and GPx were measured by ELISA. Flow cytometry (FACS) was employed to detect the percentages of ROS and proliferation rates. mRNA and protein expression of antiapoptotic genes (SURVIVIN and BCL2), apoptotic genes (CASPASE-3 and CASPASE-9), and MT1 and its downstream genes (JNK1, PCNA, AMPK) were tested by qPCR and western blotting. MT1 siRNA and related antagonists were used to assess the mechanism.
\end{abstract}

Results: fMSC treatment prevented cyclophosphamide (CTX)-induced follicle loss and recovered sex hormone levels. Additionally, fMSCs significantly decreased oxidative damage, increased oxidative protection, improved antiapoptotic effects, and inhibited apoptotic genes in vivo and in vitro. Furthermore, fMSCs also upregulated MT1, JNK1, PCNA, and AMPK at the mRNA and protein levels. With MT1 knockdown or antagonist treatment in normal hGCs, the protein expression of JNK1, PCNA, and AMPK and the percentage of proliferation were impaired.

Conclusions: fMSCs might play a crucial role in mediating follicular development in the POI mouse model and stimulating the activity of POI hGCs by targeting MT1.

Keywords: Fetal mesenchymal stem cells, Premature ovarian insufficiency, Reactive oxygen species, MT1

\section{Background}

Primary ovarian insufficiency (POI) or premature ovarian failure (POF) is a heterogeneous gynecological endocrine disorder. Cessation of ovarian function, followed by abnormal levels of gonadotropins and estrogen, are the characteristics of POI, which affects $4 \%$ of women under the age of

\footnotetext{
* Correspondence: huangboxiannj@163.com; hongliszivf@163.com

†Boxian Huang, Chunfeng Qian and Chenyue Ding contributed equally to this work.

${ }^{1}$ Center of Reproduction and Genetics, Affiliated Suzhou Hospital of Nanjing Medical University, Suzhou Municipal Hospital, Suzhou 215002, China
} Full list of author information is available at the end of the article
35 years old $[1,2]$. The cause of POI is not fully understood; however, the decreased quality and quantity of follicles and oocytes in POI may be affected by abnormal genetic factors, iatrogenic factors (chemotherapy and radiotherapy process), metabolic factors, mitochondrial dysfunction, autoimmunity and environmental factors $[3,4]$. Due to the mysterious and complicated nature of POI, there are a variety of approaches to treating POI, including hormonal replacement therapy, melatonin supplementation, dehydroepiandrosterone (DHEA) supplementation, immunomodulatory therapy, and stem cell therapy [5].

(c) The Author(s). 2019 Open Access This article is distributed under the terms of the Creative Commons Attribution 4.0 International License (http://creativecommons.org/licenses/by/4.0/), which permits unrestricted use, distribution, and 
Given that hormone replacement therapy can only relieve menopausal symptoms, stem cell therapy provides a promising therapeutic approach to preserve fertility for POI patients who want to have a healthy baby. MSCs derived from bone (BMSCs) [6], adipose-derived stem cells (ADSCs) [7], human menstrual-derived stem cells [8], and perinatal stem cells (amniotic fluid, amniotic membrane) $[9,10]$, transplanted into a POI/POF animal model via intraperitoneal injection, intravenous injection, or microinjection needles at laparotomy to rescue ovarian function. In addition, conditioned medium of human amniotic epithelial cells (hAECs) [11] and exosomes from ADSCs [12] cocultured with human POI ovarian granule cells recovered ovarian function by promoting the proliferation rate and inhibiting the apoptosis rate. Moreover, some researchers showed that estrogen-sensitive epithelial cells [13] and granulose-like cells derived from human induced pluripotent stem cells (iPSCs) could rescue ovarian function [14].

Using healthy and functional cells to replace aged or damaged cells is the aim of stem cell therapy, and stem cells need to maintain cell viability, properties, and function before and after implantation in vivo. Some studies have indicated that fetal MSCs have longer lifespans than adult MSCs when they express similar immunomodulatory properties [15-17]. fMSCs from first trimester livers express many pluripotency stem cell markers and have greater telomerase activity than that of adult MSCs [18]. Recently, research has indicated that fetal liver (FL) MSC-derived exosomes might be a powerful immunomodulator to inhibit proliferation, activation, and cytotoxicity in NK cells through exosome-associated TGF [19]. Moreover, fetal MSCs have been detected in the bone marrow, blood, and liver in early gestation [18]. Compared to the fetal blood and bone marrow, fMSCs of the liver are easily obtained as a promising candidate. However, whether fMSCs of the liver improve ovarian fertility preservation and the potential molecular mechanisms are not clear.

Although almost all of the studies on stem cell therapy show the efficacy of POI treatment, the underlying molecular mechanism and safety are not fully understood. The possible therapeutic mechanisms are involved in migration, antiapoptotic effects, antifibrotic activity, anti-inflammation, immunoregulation, and antioxidative stress [7]. An accumulating body of data indicates that melatonin has been discovered to mediate reproduction through interactions with melatonin receptor type 1 (MT1) and type 2 (MT2) in ovaries [20-22]. Melatonin combines with MT1 and MT2 to reduce levels of reactive oxygen species (ROS) [23, 24], increase glutathione S-transferase (GST) and glutathione peroxidase (GPx) activities, and inhibit glutathione (GSH) [25] and plasma Se levels [26]. A previous study showed that melatonin inhibited apoptosis and increased MT2, superoxide dismutase (SOD), and GPx4 expression, and an antagonist of MT1 and MT2 blocked these protective effects in follicular atresia and porcine GCs [27]. Another study reported that knockdown of MT1 in mouse GCs upregulated apoptosis and impeded proliferation. However, continuous follicle-stimulating hormone (FSH) administration inhibited apoptosis and improved proliferation observably [28]. Moreover, a recent study detected that melatonin had antioxidant effects that mediate cytoprotective activity against cisplatin-induced ovary damage by the MT1 receptor [29]. Although the reproductive protection role of melatonin has been widely explored in various animal models, these studies have focused on melatonin and its relevant receptors. Therefore, it is still necessary to evaluate whether other materials such as fMSCs can regulate the MT1 receptor, which could maintain follicle number and sex hormone levels by changing the level of ROS, related antioxidant enzyme activity, and the balance between apoptotic and antiapoptotic activities in the ovary.

Therefore, the objectives of this study were to evaluate whether fMSCs could restore ovarian function in POI mice, whether MT1 is involved in protective regulation in POI mice and POI hGCs, and whether the potential ovary protective effects of fMSCs are targeted to MT1.

\section{Methods \\ Isolation and culture of human fetal mesenchymal stem cells (fMSCs)}

Fetal liver tissue collection for research purposes was approved by the Research Ethics Board of Suzhou Hospital Affiliated with Nanjing Medical University. Tissues were obtained from legally aborted healthy first trimester fetuses ( $n=10$, range from 10 to 12 weeks). All the donors with written consent for the use of fetal tissue screened negative for hepatitis virus, syphilis, and human immunodeficiency virus.

Human first trimester fMSCs from the livers were isolated and cultured as described previously with some modification [30]. In brief, the fresh fetal liver was collected, minced, and filtered through a nylon filter with a size of $70 \mu \mathrm{m}$ (BD, USA). The single cells were resuspended in culture medium that included DMEM (Thermo, USA), 10\% fetal bovine serum (Thermo, USA), $2 \mathrm{mM}$ L-glutamine, $50 \mathrm{U} / \mathrm{ml}$ penicillin, and $50 \mathrm{mg} / \mathrm{ml}$ streptomycin (Thermo, USA). Cells were plated into 6well plates at a density of $10^{5}$ cells $/ \mathrm{cm}^{2}$ and incubated in a regular incubator. After $72 \mathrm{~h}$, floating cells were cleared, and the medium was changed every other day. When the cells grew to $80-90 \%$ confluence, they were digested with $0.25 \%$ trypsin and $1 \mathrm{mM}$ EDTA (Thermo, USA) at $37^{\circ} \mathrm{C}$ for $5 \mathrm{~min}$ for the experiments. The fMSCs were confirmed to express the MSC markers (CD105, 
CD29, CD73, and CD90) using flow cytometry. The pluripotency stem cell markers Oct-4, Nanog, and Rex-1 were tested by western blot, and the differentiation ability of adipogenesis, chondrogenesis, and osteogenesis was determined. Third- to fourth-passage fMSCs were used for the experiments.

\section{Isolation and culture of primary human granulose cells (hGCs)}

Primary hGCs were isolated from POI patients and young tubal factor infertility patients (age $<40$ years old) undergoing oocyte retrieval for in vitro fertilization as described in our study [12]. Briefly, follicular fluid was collected for research purposes and contained discarded red blood cells and granulosa cells. These cells were removed by centrifugation, and the GCs were purified using density centrifugation with Percoll solution (Sigma, USA). Cell culture medium included DMEM/ F12 media (Thermo, USA), 10\% fetal bovine serum (Thermo, USA), $50 \mathrm{mg} / \mathrm{ml}$ streptomycin, $50 \mathrm{U} / \mathrm{ml}$ penicillin (Thermo, USA), $1 \times$ GlutaMAX (Thermo, USA), and $100 \mathrm{mg} / \mathrm{ml}$ streptomycin sulfate (Thermo, USA).

\section{Experimental animals}

Female ICR mice $(n=150)$, aged from 7 to 8 weeks, were obtained from Nanjing Medical University. Three groups were divided randomly. The first group was the normal group (NG) $(n=50)$ and received no treatment. The second group, the POI group $(n=50)$, received a single intraperitoneal (i.p.) injection of cyclophosphamide (CTX, Sigma-Aldrich, $120 \mathrm{mg} / \mathrm{kg}$ injected everyday continued for 2 weeks). The third group, the fMSC group $(n=50)$, received a tail vein injection of approximately $1 \times 10^{6}$ fMSCs 2 weeks after CTX injection. The feeding environment and daily inspection of the mice were the same as in our previous study [12]. The blood was collected at $0,1,2,3$, and 4 weeks after the treatment with fMSCs synchronously in the three groups. $0.5 \mathrm{ml}$ blood samples were obtained by retroorbital puncture under anesthesia. After being centrifuged at $4000 \mathrm{r} / \mathrm{min}$ for $10 \mathrm{~min}$, blood samples were incubated at room temperature for $1 \mathrm{~h}$. The blood and ovaries were used for ELISA, histological, FACS, qPCR, and western blot analyses.

Adipogenic, osteogenic, and chondrogenic differentiation As previously described [10], fMSCs were incubated in human mesenchymal stem cell differentiation kits (adipogenesis, osteogenesis, and chondrogenesis differentiation kits, Thermo Fisher, USA). According to the instructions, adipogenesis was detected by Oil red $\mathrm{O}$ solution after 14 days of culture, osteogenesis was tested by the alizarin red dye method after 28 days of culture, and an Alcian blue dye assay was used to test chondrogenesis after 21 days of culture.

\section{Histological analysis}

The three groups of mice ( $n=10$ per group) were euthanized synchronously at 8 weeks after treatment with fMSCs. Ovaries were harvested and fixed in $10 \%$ formalin. After $24 \mathrm{~h}$ of fixation, increasing concentrations of ethanol dehydrated the ovarian tissue, xylene clarified the ovarian tissue, and the tissues were paraffinembedded. The paraffin was sectioned ( $5 \mu \mathrm{m}$ thick) for $H \& E$ staining to observe the morphological structure of the mouse ovaries. Four hierarchical follicles were classified using the accepted morphological criteria [31]. In each ovary, three representative sections were used to count the number of antral follicles and total follicles. Only follicles containing an oocyte were counted.

\section{ELISA analysis}

Plasma from the three groups of mice was collected at 0 , $1,2,3,4$, and 8 weeks after fMSC treatment. An ELISA kit (Mybiosource, USA) was employed to detect the levels of E2, AMH, and FSH according to the directions. In addition, mouse plasma and hGCs (NG, POI, and fMSCs) with or without fMSC treatment were also harvested to evaluate the levels of malondialdehyde (MDA), lactate dehydrogenase $(\mathrm{LDH}), \mathrm{SOD}$, glutathione reductase (GR), catalase (CAT), and GPx using an ELISA kit (Cayman Chemical, USA). In brief, the test plate containing $50 \mu \mathrm{l}$ serum or supernatant sample per well was wrapped and incubated at $37^{\circ} \mathrm{C}$ for $30 \mathrm{~min}$. Then, a wash buffer was used to wash the dried wells five times (10 s per wash). Thereafter, $50 \mu \mathrm{l} \mathrm{HRP-conjugate} \mathrm{reagent}$ was added to each well and incubated at $37^{\circ} \mathrm{C}$ for 60 min. Wash buffer was used to wash the wells five times (10 s, per wash). Then, $50 \mu \mathrm{l}$ of substrate A solution and substrate B solution were mixed together at $37^{\circ} \mathrm{C}$ for 15 min. Then, $50 \mu \mathrm{l}$ of stop solution was added to each well. Finally, a spectrophotometer (BioTek, USA) was used to test the light absorbance.

\section{MT1 gene silencing with RNA interference or the antagonist luzindole}

To silence the MT1 gene, MT1 siRNA (MyBioSource, USA) was transfected into normal hGCs as previously described [12, 28]. There were three groups in this study. The first group was normal hGCs without any treatment. The second group, the normal hGCs, was transfected with MT1 siRNA (hGCs-MT1 ${ }^{\mathrm{KD}}$ ). The third group was hGCs$M T 1^{\mathrm{KD}}$ cells treated with fMSCs. Briefly, fMSCs were seeded on the upper coculture transwell inserts, while hGCs-MT1 ${ }^{\mathrm{KD}}$ cells were on the bottom of a six-well culture plate (Corning, USA). In brief, the hGCs were seeded in three 6-well plates $72 \mathrm{~h}$ prior to transfection, and the confluency reached 50-60\%. MT1 siRNA was transfected into hGCs using Dharmafect 1 (GE Healthcare, USA). Fresh culture medium was added after transfection for 6 
$h$, and the hGCs were used for subsequent experiments after $72 \mathrm{~h}$. The efficiency of MT1 gene silencing was verified by western blot analysis.

In addition, the pharmacological modulation of MT1 was analyzed by the administration of the MT1 antagonist luzindole. Briefly, normal hGCs were treated with luzindole at different final concentrations $(10 \mu \mathrm{M}, 20 \mu \mathrm{M}$, $40 \mu \mathrm{M}$, and $80 \mu \mathrm{M})$ for 2 weeks. The inhibitory effect of MT1 was verified by western blotting. Administration of $40 \mu \mathrm{M}$ and $80 \mu \mathrm{M}$ luzindole efficiently blocked the protein expression of MT1. Therefore, three groups were divided randomly. The first group, the NG group, included normal hGCs without treatment. The second group included normal hGCs treated with luzindole at a final concentration of $40 \mu \mathrm{M}$. The third group included normal hGCs treated with luzindole and fMSCs together. fMSCs were seeded on the upper coculture inserts, while hGCs were seeded on the bottom of a six-well culture plate using a transwell system (Corning, USA).

\section{Flow cytometry analysis}

fMSCs were dissociated with $0.05 \%$ trypsin-EDTA at $37^{\circ} \mathrm{C}$ for $5 \mathrm{~min}$. The ovary tissues were dissected, washed in PBS, and then enzymatically digested to single-cell suspensions ( $0.25 \%$ trypsin-EDTA for $15 \mathrm{~min})$. Single-cell suspensions were passed through a $70-\mu \mathrm{m}$ nylon filter (BD, USA). After washing with PBS, the collected cells were suspended in buffer (0.1\% BSA in PBS). To analyze cell surface markers on fMSCs, the dissociated cells were stained with the following PE-conjugated antibodies: antiCD105, anti-CD29, anti-CD73, anti-CD90, anti-CD34, and anti-CD45, all purchased from Becton Dickinson and Company (USA). The hGCs and cell suspensions from ovarian tissues were stained with anti-KI67 (BD, USA), anti-ROS (Abcam, USA), or its corresponding isotype control at $4{ }^{\circ} \mathrm{C}$ for $30 \mathrm{~min}$. The stained cells were analyzed with a flow cytometer (Beckman, USA) using the manufacturer's directions.

\section{RNA extraction and real-time polymerase chain reaction (qPCR)}

Total RNA was extracted from the hGCs and ovarian tissues by using the RNeasy mini kit (QIAGEN, USA) and reverse-transcripted to cDNA by using the PrimeScript RT reagent kit (Takara, Japan) as described previously [12]. qPCR on amplified cDNA was performed with Power SYBR Premix Ex Taq (Takara, Japan) using a Thermal Cycler Dice Real Time System (Takara, Japan). The data were analyzed by the 2- $\Delta \Delta \mathrm{Ct}$ calculation method with glyceraldehyde 3phosphate dehydrogenase (GAPDH) as the internal reference. The primer sequences used are listed in Additional file 1: Table S1.

\section{Western blot analysis}

fMSCs, hGCs, and ovarian tissues were harvested for protein extraction. Western blotting was performed as previously described [12]. The primary antibodies used for fMSCs were anti-Oct4, anti-Nanog, anti-Rex1, and anti- $\beta$-Actin, all purchased from Abcam (USA). The primary antibodies used for hGCs and the ovarian tissues were anti-SURVIVIN, anti-BCL2, anti-CASPASE-3, antiCASPASE-9, anti-MT1, anti-JNK1, anti-PCNA, antiAMPK, anti- $\beta$-Actin, and anti-GAPDH, all purchased from Abcam (USA).

\section{Statistical analysis}

All results are expressed as the means \pm SD. SPSS 19.0 software was used to carry out one-way ANOVA, with $p<0.05$ considered statistically significantly different.

\section{Results}

\section{Characterization of $\mathrm{fMSCs}$}

To confirm whether the cells from first trimester fetal livers have properties of MSCs, western blot, cell differentiation, and FACS analysis were performed. The differentiation potency of these cells was confirmed by adipogenesis, osteogenesis, and chondrogenesis differentiation (Fig. 1a). Then, the various immunophenotypes of the cells were detected by FACS. As shown in Fig. 1b, adherent fetal liver cells were highly expressed in the MSC markers CD105 (97.3\%), CD29 (77.2\%), CD73 (98.1\%), and CD90 (99.0\%) and expressed low levels of the hematopoietic markers CD34 (23.2\%) and CD45 (20.5\%). In addition, western blot assay indicated that the cells expressed the transcription factor markers Oct4, Nanog, and Rex-1 (Fig. 1c).

\section{Effects of fMSCs on follicle numbers and sex hormone levels in a CTX-induced POI mouse model}

To better understand the potential therapeutic effects of fMSCs, we compared the number of follicles and the serum levels of sex hormones in mice in different treatment groups. The number of antral follicles in the fMSC group was significantly increased in comparison to that in the POI group after treatment 8 weeks (Fig. 2a). The total follicle numbers exhibited similar results (Fig. 2a). Then, ELISA was used to analyze the levels of E2, AMH, and FSH in serum from the three groups at $0,1,2,3,4$, and 8 weeks after fMSC injection. Compared to levels in the POI group, the levels of E2 and AMH in the fMSC group were elevated similar to those in the NG group in a time-dependent manner (Fig. 2c, d). The level of FSH showed a trend toward a time-dependent decrease in stimuli compared to normal levels (Fig. 2e). 


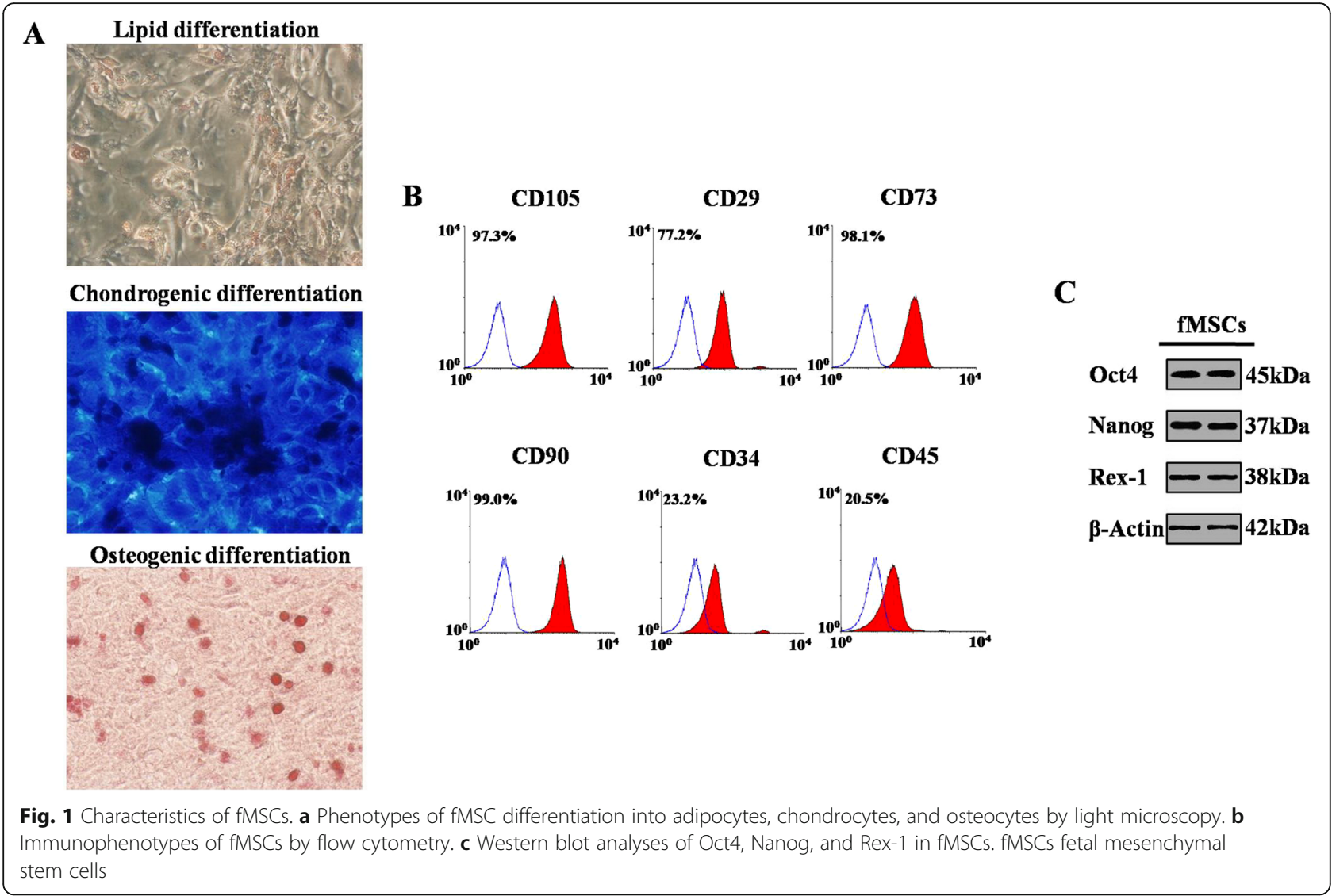

fMSCs decreased oxidative damage, increased oxidative protection, improved antiapoptotic effects, and inhibited apoptosis in POI hGCs

Given that oxidative stress is an inducer of pathological ovarian aging [32], we speculated that the fMSCmediated improvement in ovarian activity might be attributed to the decreased ROS level. To test this hypothesis, POI hGCs were treated with $\mathrm{fMSCs}$ to measure the ROS rate by FACS (Fig. 3a). The ROS rate in the fMSC group (14.0\%) was remarkably suppressed compared with that in the POI group (86.5\%) as shown in Fig. 3b. We also measured oxidative and antioxidative enzymes and metabolite activity to further confirm the influence of ROS. ELISA was used to quantitatively evaluate MDA, LDH, SOD, GR, CAT, and GPx activities. The activities of SOD, GR, CAT, and GPx in the POI group were decreased significantly, whereas the activities of MDA and LDH were elevated markerly which compared to the NG group. All these enzymes and metabolites were restored nearly to the level of the NG group after fMSC treatment with POI hGCs (Fig. 3c-h).

Thereafter, qPCR was employed to detect the mRNA expression levels of antiapoptotic and apoptotic markers in hGCs in the three groups (NG, POI, and fMSC treatment). At the mRNA level, a more than fourfold decrease in antiapoptotic markers (SURVIVIN and BCL2) was observed in the POI group in comparison to that in the NG group (Fig. 3i), while a more than threefold increase in apoptotic markers (CASPASE-3 and CASPASE-9) was observed in the POI group in comparison to that in the NG group (Fig. 3j). However, these same markers in the fMSC group were rescued nearly to the level of the NG group (Fig. 3i-j). Moreover, a similar trend in protein expression was exhibited in the three groups. fMSCs upregulated antiapoptotic genes and downregulated apoptotic genes at the protein level (Fig. 3k).

fMSCs decreased oxidative damage, increased oxidative protection, improved anti-apoptosis levels, and inhibited apoptosis levels in a CTX-induced POI mouse model Because of the in vitro effects of $\mathrm{fMSCs}$ on oxidation resistance, anti-apoptosis promotion, and apoptosis inhibition, fMSCs were transplanted into a CTX-induced POI mouse model (Fig. 4a). In line with our results, fMSCs significantly decreased the ROS rate (16.3\%) in POI mouse ovaries compared to that of the POI group (78.2\%) as shown in Fig. $4 \mathrm{~b}$. In addition, the activities of oxidoreductases (MDA and $\mathrm{LDH}$ ) and antioxidases (SOD, GR, CAT, and GPx) were assessed in the POI group. After fMSC transplantation into POI mouse ovaries, the levels of MDA and $\mathrm{LDH}$ were downregulated, and the levels of SOD, GR, CAT, and GPx were upregulated rapidly (Fig. $4 \mathrm{c}-\mathrm{h}$ ). qPCR and western 


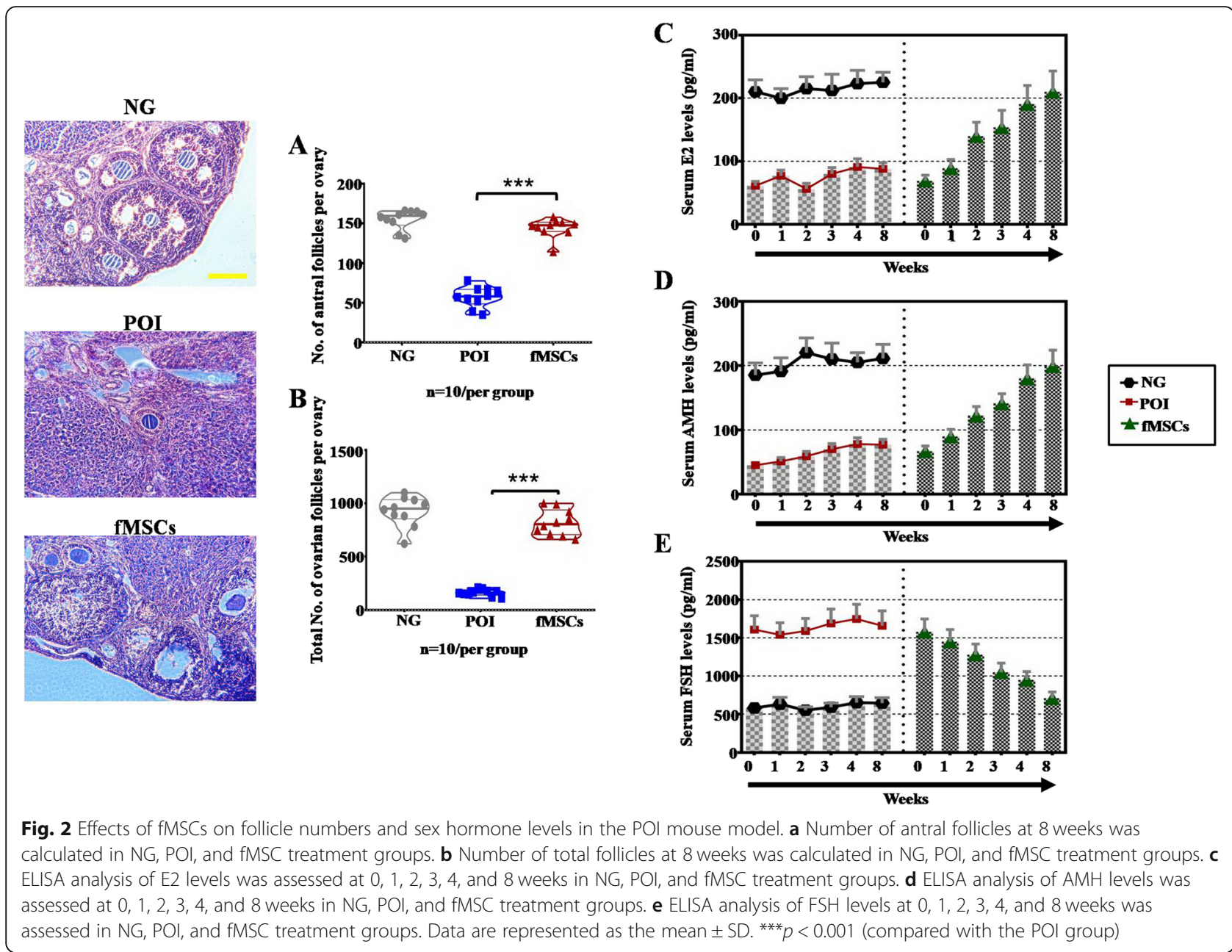

blot analyses also confirmed that the fMSCs improved the antiapoptotic genes (SURVIVIN and BCL2) and repressed the apoptotic markers (CASPASE-3 and CASPASE-9) at the mRNA (Fig. $4 \mathrm{i}, \mathrm{j}$ ) and protein levels in ovaries of POI mice (Fig. 4k).

\section{fMSCs upregulated MT1, JNK1, PCNA, and AMPK in POI} hGCs and the CTX-induced POI mouse model

To further explore the potential molecular mechanism, we selected MT1, c-Jun N-terminal kinase 1 (JNK1), proliferation cell nuclear antigen (PCNA), and AMP-activated protein kinase (AMPK) as candidate target genes for fMSCs to improve ovarian function. qPCR analysis was performed to determine the expression of the MT1, JNK1, PCNA, and AMPK genes in the three groups (NG, POI, and fMSCs) in vitro. The mRNA expression levels of MT1, JNK1, PCNA, and AMPK in the fMSC treatment groups were significantly upregulated similar to the levels in the NG group (Fig. 5a). The mRNA expression level of these genes was also elevated significantly in the fMSC group in vivo (Fig. 5c).
In addition, western blot analysis was used to detect the protein expression levels of MT1, JNK1, PCNA, and AMPK in vivo and in vitro. In the POI hGC group, the protein expression levels of MT1, JNK1, PCNA, and AMPK were less than half that in the NG group (Fig. 5b). After incubation with POI hGCs and injection into the POI mouse model, western blot analysis showed that fMSCs remarkably upregulated MT1, JNK1, PCNA, and AMPK expression ex vivo (Fig. 5b) and in vivo (Fig. 5d).

\section{Therapeutic effect of $\mathrm{fMSCs}$ was displayed by targeting MT1}

Given that melatonin exerts numerous biological functions for ovarian improvement via the MT1 and MT2 receptors of the G-protein-coupled superfamily, the molecular mechanism of MT1 was further evaluated by MT1 siRNA transfection and MT1 antagonist luzindole treatment. The knockdown efficiency of MT1 was detected by using western blotting. After MT1 was knocked down in normal hGCs, the low MT1 level was tested as shown in Fig. 6a. The results indicated that hGCs-MT1 ${ }^{\mathrm{KD}}$ cell lines were established successfully. To explore the regulatory role of 


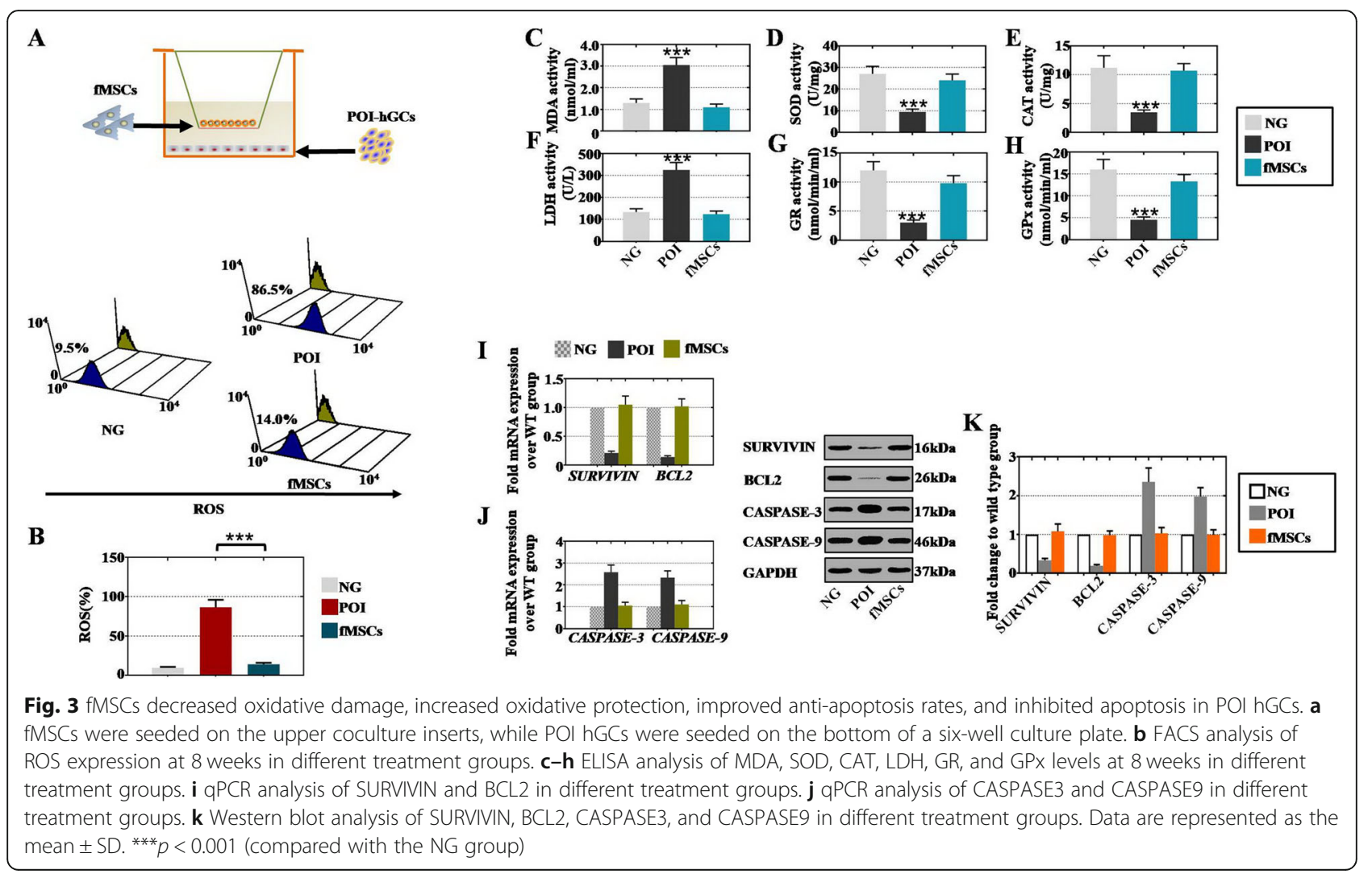

MT1 in the proliferation of hGCs, incubation of fMSCs with the MT1 $1^{\mathrm{KD}} \mathrm{hGCs}$ was utilized to detect the efficacy in the present experiment. Western blot analysis revealed that JNK1, PCNA, and AMPK genes at the protein level were downregulated in the $\mathrm{MT}^{\mathrm{KD}}$ group, whereas there were no significant changes after hGCs-MT1 ${ }^{\mathrm{KD}}$ coculture with fMSCs (Fig. 6b). To further investigate the MT1 mechanism, the MT1 antagonist luzindole at different concentrations $(10 \mu \mathrm{M}, 20 \mu \mathrm{M}, 40 \mu \mathrm{M}$, and $80 \mu \mathrm{M})$ was exogenously supplemented into normal hGCs. As Fig. 6c shows, the administration of $40 \mu \mathrm{M}$ and $80 \mu \mathrm{M}$ antagonist blocked the protein expression of MT1 efficiently. Therefore, the expression levels of JNK1, PCNA, and AMPK were examined at a concentration of $40 \mu \mathrm{M}$, and our results showed that fMSCs did not recover gene expression after antagonist treatment (Fig. 6d).

In addition, we further explored whether fMSCs improved the proliferation rate of hGCs-MT1 ${ }^{\mathrm{KD}}$. FACS analysis indicated that the percentage of $\mathrm{Ki}-67^{+}$hGCs was sharply decreased to $24.1 \%$ in the $\mathrm{MT}^{\mathrm{KD}}$ group, whereas the percentage was $89.5 \%$ in the NG group (Fig. 6e). The percentage of $\mathrm{KI} 67^{+}$hGCs was slightly upregulated to $45.9 \%$ in the MT1 ${ }^{\mathrm{KD}} / \mathrm{fMSCs}$ group as shown in Fig. $6 \mathrm{e}$.

\section{Discussion}

Due to infertility, POI causes a feeling of sadness and depression in many couples and families. The main pathogenesis of POI disease is elaborated in depth, which is convenient for treatment. However, the etiology of POI remains unclear in the large majority of cases. Although almost all of the studies point to the efficacy of stem cells in POI treatment, the potential mechanisms are not fully understood. The findings from this study demonstrate that fMSC treatment attenuates ovarian damage in a CTX-induced POI mouse model and promotes proliferation of POI hGCs by regulating MT1. To our knowledge, this is the first study to investigate the interaction between fMSCs derived from the liver and POI. The beneficial effects of fMSCs in restoring ovarian function in POI and the potential mechanisms are discussed below.

Although MSCs derived from various cellular sources make them attractive for treating POI disease [6-8], there is no report that discusses the therapeutic effect of MSCs derived from the fetal liver on POI treatment. The current investigation of this effect showed that the CTX-induced POI mouse model treated with fMSCs not only improved the antral follicles and total follicle numbers at four stages but also increased the sex hormone levels to nearly the normal levels (Fig. 2). The disorder of oxidative stress has been implicated in the causal pathways for POF [33,34]. The generation of ROS plays a role in cellular metabolism under normal physiological conditions, whereas increased ROS levels directly oxidize 


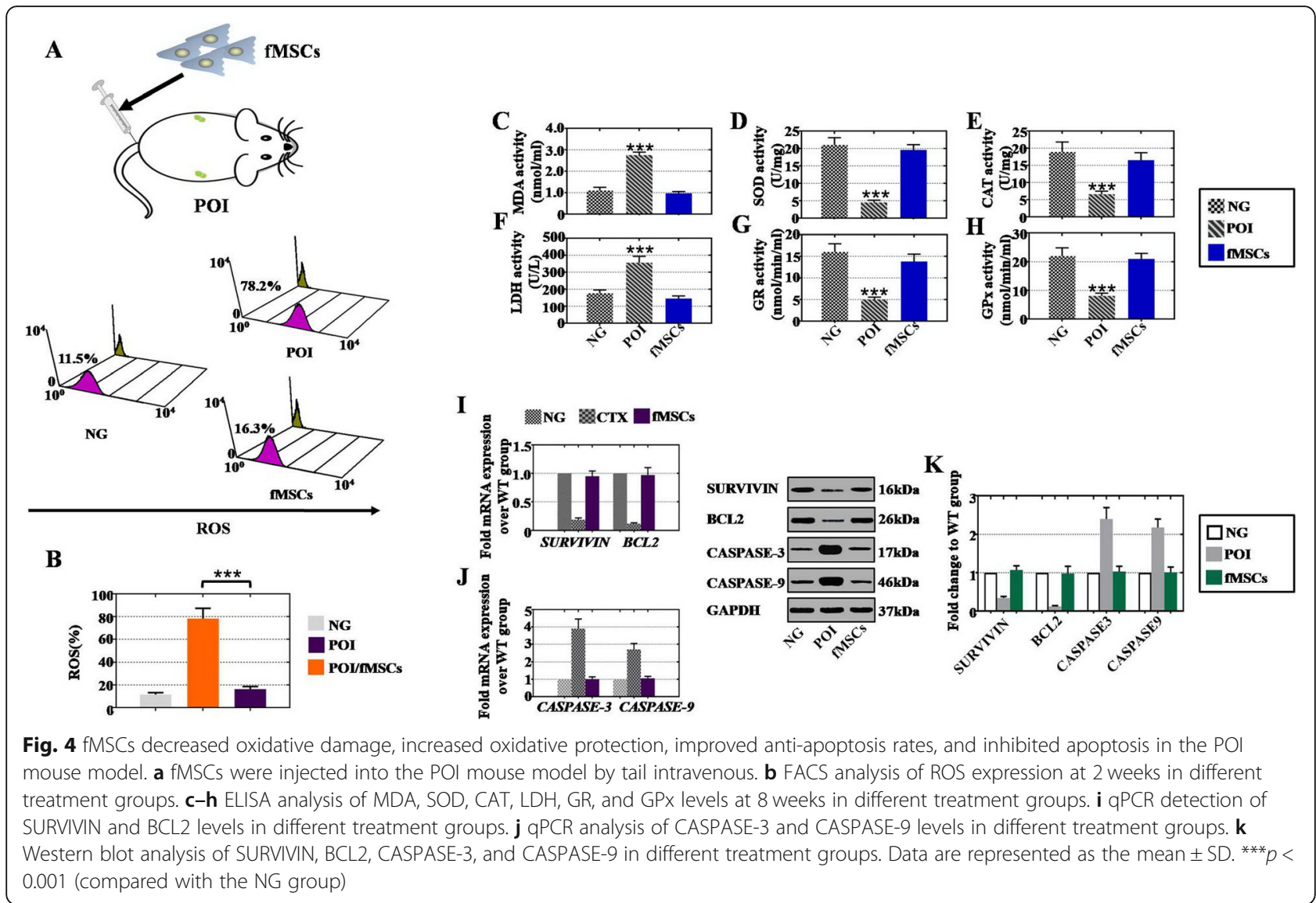

protein and DNA or indirectly activate intracellular signaling pathways that induce cellular damage [35-37]. In this study, we found that the ROS level was downregulated in the POI mouse model and POI hGCs after treatment with fMSCs (Figs. 3 and 4). LDH, a key regulator of glycolysis, and MDA, a major end product of membrane lipid peroxidation, were increased in vivo and in vitro in the POI model, while they were decreased after fMSC treatment (Figs. 3 and 4). The activities of SOD, GR, CAT, and GPx were inhibited observably in the in vivo and in vitro POI models, but they were elevated after the fMSC treatment (Figs. 3 and 4). These findings suggest that multitargeted antioxidant properties of fMSCs might defend against ovarian damage from oxygen radicals. Although stem cell therapy improves ovarian function in POI by mediating oxidative stress, which has not been reported, BMSCs did have an effective therapeutic function in colitis disease by suppressing oxidative stress [38], and melatonin effectively protected ovarian function against cisplatin-induced ovarian damage by reducing apoptosis, ROS production, and mitochondrial damage [29].

Oxidative stress has been considered an important apoptosis mediator, and the fate of ovarian cells is ultimately determined by the homeostasis of pro- and antiapoptotic genes [39]. Highly precise apoptotic and antiapoptotic regulation are involved in intrinsic and extrinsic pathways $[40,41]$. The BCL2 family plays a vital role in the intrinsic pathway [42]. Apoptosis and antiapoptotic proteins assemble and enter the mitochondria, triggering the release of cytochrome $\mathrm{c}$ to activate CASPASE-9 and CASPASE-3, which causes DNA damage and cell apoptosis [43]. The current study demonstrated that the apoptotic markers BCL2 and SURVIVIN were upregulated and the antiapoptotic markers CASPASE-3 and CASPASE-9 were downregulated in the POI mouse model and in POI hGCs treated with fMSCs (Figs. 3 and 4). In line with our findings, BMSCs resisted the apoptosis of GCs by increasing the cmyc gene and decreasing the $\mathrm{p} 21$ and bax genes to improve ovarian morphology and function in POF rats [44], and umbilical cord MSCs inhibited apoptosis of POI rat ovaries by decreasing CASPASE-3 to restore ovarian function and structure [45]. These findings suggest that fMSCs might improve fertility preservation in POI disease by improving the antiapoptotic level and inhibiting apoptosis.

Many signaling molecules, receptors, and pathways participate in the recovery of ovarian function. Currently, a growing body of evidence shows that melatonin and MT1 improve oocyte and embryo early development and regulate the level of sex hormones [22,46, 47]. JNK is a part of 

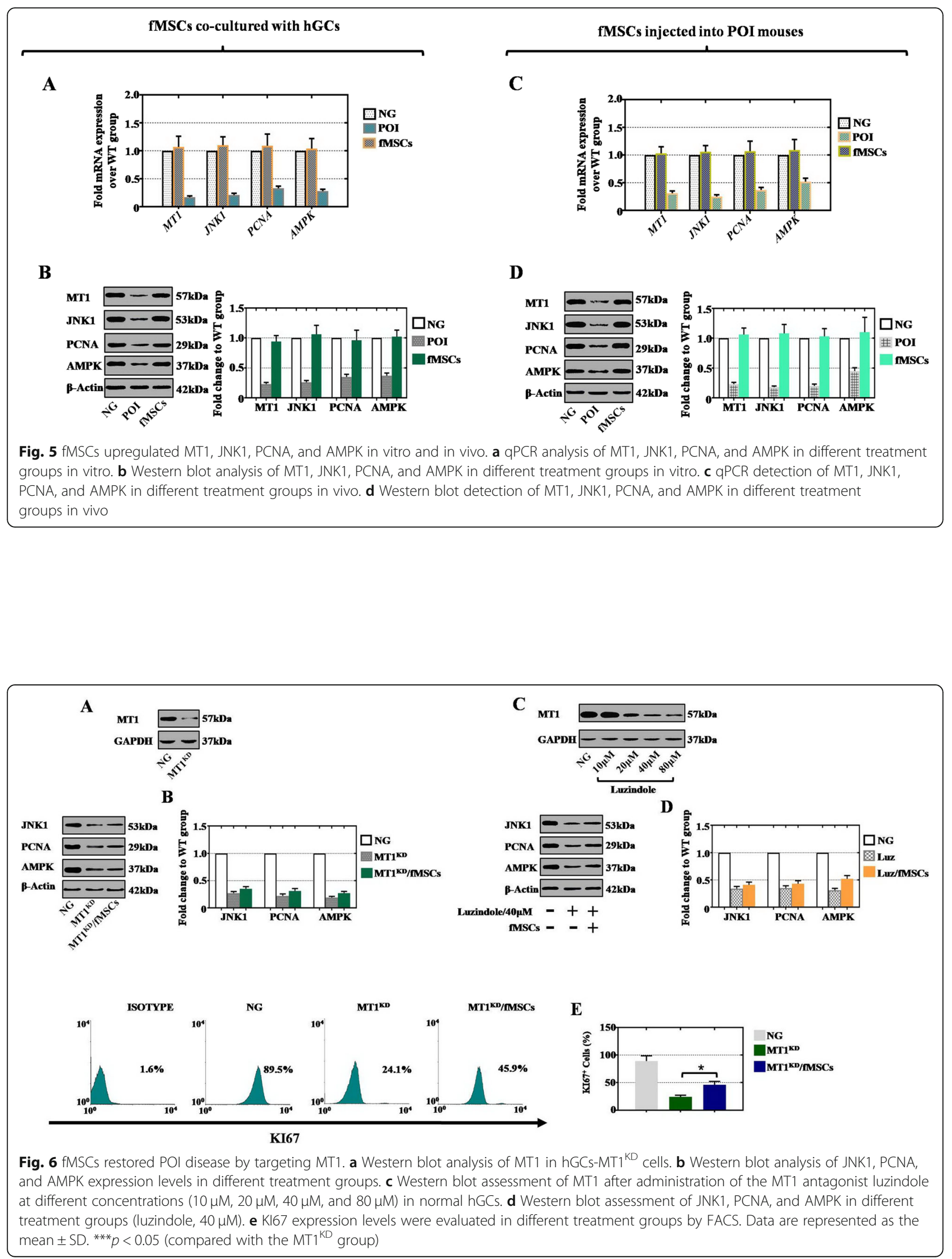
the mitogen-activated protein kinase (MAPK) signaling pathway mediating cellular responses to various stress stimuli, and its subfamily includes the JNK1, JNK2, and JNK3 genes [48]. JNK modulates a variety of biological functions, including proliferation, apoptosis, migration, differentiation, and inflammation [49-51]. PCNA is the major regulator of replication fidelity and DNA damage repair, and it is essential for normal cellular proliferation [52]. AMPK is a vital intracellular energy sensor that promotes catabolic pathways to generate more ATP, suppresses anabolic signaling in low ATP conditions, and mediates the coordination of cell growth and metabolism $[53,54]$. In this study, we observed a significant decrease in the expression of MT1, JNK1, PCNA, and AMPK by qRCR and western blotting in the POI mouse model and POI hGCs, whereas these genes were markedly increased after treatment with fMSCs (Fig. 5). Together, these data highlight that MT1, JNK1, PCNA, and AMPK may participate in cellular proliferation, apoptosis, migration, and ovary damage. Although stem cell therapy restores ovarian function in POI by directly mediating MT1, JNK1, PCNA, and AMPK, which has not been previously reported, a supporting study revealed that melatonin effectively elevated the percentage of PCNA-positive granulosa and theca cells [29] and that the radiation-POF mouse model treated with niacin highly expressed PCNA in the GCs [55]. In a recent study, human ovaries and hGCs were exposed to cisplatin in vitro, which elevated the levels of the phosphorylated forms of SAPK/JNK and triggered apoptosis [56]. A previous study demonstrated that MT1, AMPK, and p-AMPK were upregulated in the ovaries of melatonin-treated mice compared to those of untreated mice, which is similar to the results of our study [57].

Given that melatonin, through interaction with membrane receptors (MT1 and MT2), plays a vital role in reproduction, our aim was to explore whether cellular materials such as fMSCs can regulate the MT1 receptor to preserve the fertility of ovaries by influencing ROS and related antioxidant enzyme activity, proliferation, or apoptosis rate in the ovary niche during oogenesis. When normal hGCs inhibited MT1 expression effectively by RNA interference and chemical inhibitors, the protein levels of JNK1, PCNA, and AMPK were not markedly increased after fMSC treatment, and the percentage of cells with the proliferation marker KI67 was elevated significantly when hGCs-MT1 ${ }^{\mathrm{KD}}$ were treated with fMSCs (Fig. 6). This phenomenon may indicate that MT1 is not the only regulator of proliferation.

Our results are in agreement with an earlier study that showed that silencing of MT1 by RNA interference led to increased apoptosis in mGCs, whereas the apoptotic effect was inhibited after mGCs-MT1 ${ }^{\mathrm{KD}}$ administration with FSH [28]. Similar to our findings, a previous experiment indicated that administration of the MT1/MT2 receptor antagonist (luzindole) inhibited the therapeutic effect of melatonin on chemotoxic ovary damage [29]. We observed similar results in our study, and fMSCs were unable to increase JNK1, PCNA, and AMPK expression after inhibition of MT1 (Fig. 7).

\section{Conclusion}

In summary, fMSCs can be used to recover ovarian function. Furthermore, we explore the potential mechanisms. First, fMSCs exert antioxidant effects through their downregulation of ROS, MDA, and LDH and upregulation of SOD, GR, CAT, and GPx levels in vitro and in vivo. In addition, fMSCs display proliferation effects due to their elevation of SURVIVIN and BCL2 and repression of CASPASE-3 and CASPASE-9. Moreover, fMSCs might enhance the biological activity of hGCs by mediating MT1 and its downstream genes. These discoveries raise the possibility of using fMSCs to treat POI disease in humans. Furthermore, we elaborated the mechanism by which MT1 is a preponderant therapeutic target for restoring ovarian function.

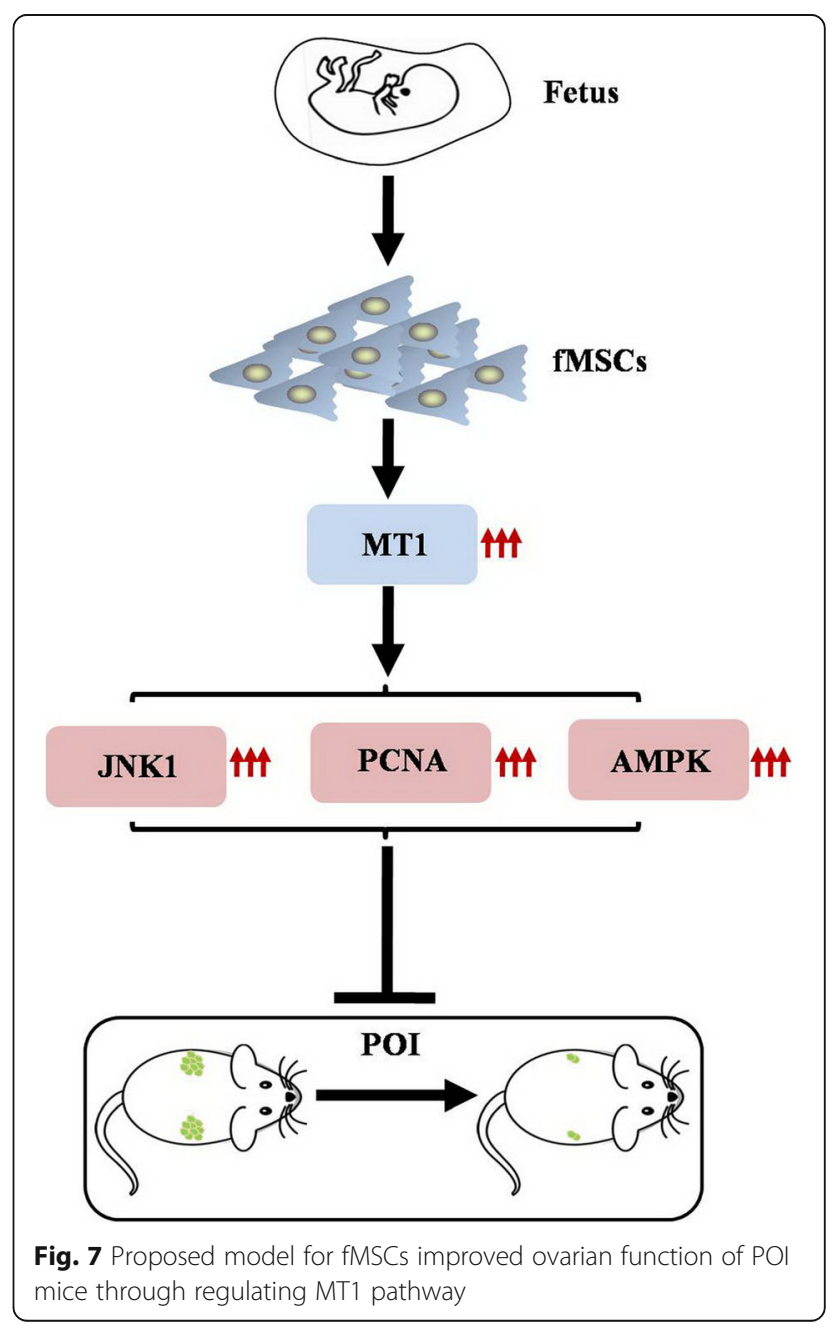




\section{Supplementary information}

Supplementary information accompanies this paper at https://doi.org/10. 1186/s13287-019-1490-8.

Additional file 1: Table S1. Designations, sequences, and the sizes of real-time PCR amplicons.

\section{Abbreviations}

POI: Premature ovarian insufficiency; POF: Premature ovarian failure: fMSC: Fetal mesenchymal stem cells; MT1: Melatonin receptor 1; NG: Normal group; CTX: Cyclophosphamide; hGC: Human granulosa cell; E2: Estradiol; AMH: Anti-Mullerian hormone; FSH: Follicle-stimulating hormone; ROS: Reactive oxygen species; MDA: Malondialdehyde; SOD: Superoxide dismutase; CAT: Catalase; LDH: Lactate dehydrogenase; GR: Glutathione reductase; GPx: Glutathione peroxidase; JNK1: c-Jun N-terminal kinase 1; PCNA: Proliferation cell nuclear antigen; AMPK: AMP-activated protein kinase; ELISA: Enzyme-linked immunosorbent assay; FACS: Flow cytometry; FACS: Fluorescence-activated cell sorting; HE: Hematoxylin and eosin; qPCR: Quantitative polymerase chain reaction; GAPDH: Glyceraldehyde 3phosphate dehydrogenase

\section{Authors' contributions}

$\mathrm{BH}, \mathrm{CQ}$, and $\mathrm{CD}$ performed the experiments and collected and assembled data. QM contributed to hGC collection, purification, and culture. YZ established the POI mouse model by CTX. BH and HL planned the project, analyzed the experiments, and reviewed and edited the paper. All authors read and approved the final manuscript.

\section{Funding}

This work was supported by the grants from National Natural Science Foundation of China $(81801515,81801494)$, Suzhou talent training program (GSWS2019005), Suzhou introduce expert team of clinical medicine (SZYJTD201708), and Suzhou Science and Technology for People's Livelihood (SYS2019097).

\section{Availability of data and materials}

All the data generated or analyzed during this study are included in this published article.

\section{Ethics approval and consent to participate}

The use of human ovarian granular cells and fetal liver was in accordance with the relevant guidelines and regulations, and the experimental protocols were approved by the Medical Ethics Committee of the Suzhou Hospital Affiliated to Nanjing Medical University (SZ-NJMU-003). All the patients provided written informed consent prior to participation in this study. Our investigation using experimental animals and ethical approval were conducted on the basis of the Nanjing Medical University Animal Center's specific guidelines and standards.

\section{Consent for publication}

Not applicable

\section{Competing interests}

The authors declare that they have no competing interests.

\section{Author details}

${ }^{1}$ Center of Reproduction and Genetics, Affiliated Suzhou Hospital of Nanjing Medical University, Suzhou Municipal Hospital, Suzhou 215002, China. ${ }^{2}$ State Key Laboratory of Reproductive Medicine, Nanjing Medical University, Nanjing 210029, China.

Received: 28 August 2019 Revised: 2 November 2019 Accepted: 6 November 2019 Published online: 29 November 2019

\section{References}

1. Coulam CB, Adamson SC, Annegers JF. Incidence of premature ovarian failure. Obstet Gynecol. 1986;67:604-6.

2. Nelson LM. Primary ovarian insuffciency. N Engl J Med. 2009;360:606-14.

3. Sukur YE, Kivancli IB, Ozmen B. Ovarian aging and premature ovarian failure. Turk Ger Gyneco Assoc. 2014;15:190-6.
4. Vujovic S. Aetiology of premature ovarian failure. Menopause Int. 2009;15:72-5.

5. Sheikhansari G, Aghebati-Maleki L, Nouri M, Jadidi-Niaragh F, Yousefi M. Current approaches for the treatment of premature ovarian failure with stem cell therapy. Biomed Pharmacother. 2018;102:254-62.

6. Mohamed SA, Shalaby SM, Abdelaziz M, Brakta S, Hill WD, Ismail N, et al. Human mesenchymal stem cells partially reverse infertility in chemotherapy-induced ovarian failure. Reprod Sci. 2018;25:51-63.

7. Ding C, Zou Q, Wang F, Wu H, Wang W, Li H, et al. HGF and BFGF secretion by human adipose-derived stem cells improves ovarian function during natural aging via activation of the SIRT1/FOXO1 signaling pathway. Cell Physiol Biochem. 2018:45:1316-32.

8. Wang $Z$, Wang $Y$, Yang $T$, Li J, Yang $X$. Study of the reparative effects of menstrual-derived stem cells on premature ovarian failure in mice. Stem Cell Res Ther. 2017;8:11.

9. Ding C, Zou Q, Wang F, Wu H, Chen R, Lv J, et al. Human amniotic mesenchymal stem cells improve ovarian function in natural aging through secreting hepatocyte growth factor and epidermal growth factor. Stem Cell Res Ther. 2018;9:55.

10. Ding $\mathrm{C}$, Li H, Wang Y, Wang F, Wu H, Chen R, et al. Different therapeutic effects of cells derived from human amniotic membrane on premature ovarian aging depend on distinct cellular biological characteristics. Stem Cell Res Ther. 2017;8:173

11. Zhang Q, Bu S, Sun J, Xu M, Yao X, He K, et al. Paracrine effects of human amniotic epithelial cells protect against chemotherapy-induced ovarian damage. Stem Cell Res Ther. 2017;8:270.

12. Huang B, Lu J, Ding C, Zou Q, Wang W, Li H. Exosomes derived from human adipose mesenchymal stem cells improve ovary function of premature ovarian insufficiency by targeting SMAD. Stem Cell Res Ther. 2018;9:216.

13. Liu T, Qin W, Huang Y, Zhao Y, Wang J. Induction of estrogen-sensitive epithelial cells derived from human-induced pluripotent stem cells to repair ovarian function in a chemotherapy-induced mouse model of premature ovarian failure. DNA Cell Biol. 2013;32:685-98.

14. Li Q, Wang S, Chen C, Zheng J. Transplantation of ovarian granulosalike cells derived from human induced pluripotent stem cells for the treatment of murine premature ovarian failure. Mol. Med. Rep. 2016;13:5053-8.

15. Lee CC, Ye F, Tarantal AF. Comparison of growth and differentiation of fetal and adult rhesus monkey mesenchymal stem cells. Stem Cells Dev. 2006;15: 209-20.

16. Lai RC, Arslan F, Tan SS, Tan B, Choo A, Lee MM, et al. Derivation and characterization of human fetal MSCs: an alternative cell source for largescale production of cardioprotective microparticles. J Mol Cell Cardiol. 2010; 48:1215-24

17. Giuliani M, Fleury M, Vernochet A, Ketroussi F, Clay D, Azzarone B, et al. Long-lasting inhibitory effects of fetal liver mesenchymal stem cells on Tlymphocyte proliferation. PLoS One. 2011;6:e19988.

18. Guillot PV, Gotherstrom C, Chan J, Kurata H, Fisk NM. Human first-trimeste fetal MSC express pluripotency markers and grow faster and have longer telomeres than adult MSC. Stem Cells. 2007;25:646-54.

19. Fan Y, Herr F, Vernochet A, Mennesson B, Oberlin E, Durrbach A. Human fetal liver mesenchymal stem cell-derived exosomes impair natural killer cell function. Stem Cells Dev. 2019;28:44-55.

20. Niles LP, Wang J, Shen L, Lobb DK, Younglai EV. Melatonin receptor mRNA expression in human granulosa cells. Mol Cell Endocrinol. 1999;156:107-10.

21. Lee CJ, Do BR LYH, Park JH, Kim SJ, Kim JK, et al. Ovarian expression of melatonin Mel1a receptor mRNA during mouse development. Mol Reprod Dev. 2001;59:126-32

22. Wang SJ, Liu WJ, Wu CJ, Ma FH, Ahmad S, Liu BR, et al. Melatonin suppresses apoptosis and stimulates progesterone production by bovine granulosa cells via its receptors (MT1 and MT2). Theriogenology. 2012;78: 1517-26.

23. Gao C, Han HB, Tian XZ, Tan DX, Wang L, Zhou GB, et al. Melatonin promotes embryonic development and reduces reactive oxygen species in vitrified mouse 2-cell embryos. J Pineal Res. 2012;52:305-11.

24. Tamura H, Takasaki A, Taketani T, Tanabe M, Kizuka F, Lee $L$, et al. The role of melatonin as an antioxidant in the follicle. J Ovarian Res. 2012;26:5.

25. Subramanian P, Mirunalini S, Dakshayani KB, Pandi-Perumal SR, Trakht I, Cardinali DP. Prevention by melatonin of hepatocarcinogenesis in rats injected with N-nitrosodiethylamine. J Pineal Res. 2007;43:305-12.

26. Navarro-Alarcon M, Ruiz-Ojeda FJ, Blanca-Herrera RM, Agil A. Antioxidant activity of melatonin in diabetes in relation to the regulation and levels of 
plasma Cu, Zn, Fe, Mn, and Se in Zucker diabetic fatty rats. Nutrition. 2013 29:785-9.

27. He Y, Deng H, Jiang Z, Li Q, Shi M, Chen H, et al. Effects of melatonin on follicular atresia and granulosa cell apoptosis in the porcine. Mol Reprod Dev. 2016;83:692-700.

28. Talpur HS, Worku T, Rehman ZU, Dad R, Bhattarai D, Bano I, et al. Knockdown of melatonin receptor 1 and induction of follicle-stimulating hormone on the regulation of mouse granulosa cell function. Reprod Biol. 2017;17:380-8

29. Barberino RS, Menezes VG, Ribeiro AEAS, Palheta RC Jr, Jiang X, Smitz JEJ, et al. Melatonin protects against cisplatin-induced ovarian damage in mice via the MT1 receptor and antioxidant activity. Biol Reprod. 2017:96:1244-55.

30. Campagnoli C, Roberts IA, Kumar S, Bennett PR, Bellantuono I, Fisk NM. Identification of mesenchymal stem/progenitor cells in human first-trimester fetal blood, liver, and bone marrow. Blood. 2001;98:2396-402.

31. Pederson T, Peters H. Proposal for a classification of oocytes and follicles in the mouse ovary. J Reprod Fertil. 1968;17:555-7.

32. Yadav AK, Yadav PK, Chaudhary GR, Tiwari M, Gupta A, Sharma A, et al. Autophagy in hypoxic ovary. Cell Mol Life Sci. 2019;76:3311-22.

33. Machi JF, Dias Dda S, Freitas SC, de Moraes OA, da Silva MB, Cruz PL, et al. Impact of aging on cardiac function in a female rat model of menopause: role of autonomic control, inflammation, and oxidative stress. Clin Interv Aging. 2016;11:341-50.

34. Venkatesh S, Kumar M, Sharma A, Kriplani A, Ammini AC, Talwar P, et al. Oxidative stress and ATPase6 mutation is associated with primary ovarian insufficiency. Arch Gynecol Obstet. 2010;282:313-8.

35. Kiffin R, Bandyopadhyay U, Cuervo AM. Oxidative stress and autophagy. Antioxid Redox Signal. 2006;8:152-62.

36. Klaunig JE, Kamendulis LM, Hocevar BA. Oxidative stress and oxidative damage in carcinogenesis. Toxicol Pathol. 2010;38:96-109.

37. Ghosh J, Das J, Manna P, Sil PC. Taurine prevents arsenic-induced cardiac oxidative stress and apoptotic damage: role of NF-kappa B, p38 and JNK MAPK pathway. Toxicol Appl Pharmacol. 2009;240:73-87.

38. Sun T, Gao GZ, Li RF, Li X, Li DW, Wu SS, et al. Bone marrow-derived mesenchymal stem cell transplantation ameliorates oxidative stress and restores intestinal mucosal permeability in chemically induced colitis in mice. Am J Transl Res. 2015;7:891-901.

39. Zhao $Y$, Zhao $H$, Zhai $X$, Dai J, Jiang $X$, Wang $G$, et al. Effects of $Z n$ deficiency, antioxidants, and low-dose radiation on diabetic oxidative damage and cell death in the testis. Toxicol Mech Methods. 2013;23:42-7.

40. Frank J, Lambert C, Biesalski HK, Thews O, Vaupel P, Kelleher DK. Intensified oxidative and nitrosative stress following combined ALA-based photodynamic therapy and local hyperthermia in rat tumors. Int J Cancer. 2003;107:941-8

41. Wyllie AH, Kerr JF, Currie AR. Cell death: the significance of apoptosis. Int Rev Cytol. 1980;68:251-306

42. Armstrong JS. Mitochondria: a target for cancer therapy. Br J Pharmacol. 2006;147:239-48.

43. Peixoto PM, Lue JK, Ryu SY, Wroble BN, Sible JC, Kinnally KW. Mitochondrial apoptosis-induced channel (MAC) function triggers a Bax/Bak-dependent bystander effect. Am J Pathol. 2011;178:48-54.

44. Guo JQ, Gao X, Lin ZJ, Wu WZ, Huang LH, Dong HY, et al. BMSCs reduce rat granulosa cell apoptosis induced by cisplatin and perimenopause. BMC Cell Biol. 2013;14:18.

45. Zheng Q, Fu X, Jiang J, Zhang N, Zou L, Wang W, et al. Umbilical cord mesenchymal stem cell transplantation prevents chemotherapy-induced ovarian failure via the NGF/TrkA pathway in rats. Biomed Res Int. 2019;2019: 6539294.

46. Sampaio RV, Conceicao S, Miranda MS, Sampaio Lde F, Ohashi OM. MT3 melatonin binding site, MT1 and MT2 melatonin receptors are present in oocyte, but only MT1 is present in bovine blastocyst produced in vitro. Reprod Biol Endocrinol. 2012;10:103

47. He C, Ma T, Shi J, Zhang Z, Wang J, Zhu K, et al. Melatonin and its receptor MT1 are involved in the downstream reaction to luteinizing hormone and participate in the regulation of luteinization in different species. J Pineal Res. 2016;61:279-90

48. Chang L, Karin M. Mammalian MAP kinase signalling cascades. Nature. 2001; 410:37-40.

49. Binetruy B, Heasley L, Bost F, Caron L, Aouadi M. Concise review: regulation of embryonic stem cell lineage commitment by mitogen-activated protein kinases. Stem Cells. 2007;25:1090-5.
50. Pearson G, Robinson F, Beers Gibson T, Xu BE, Karandikar M, Berman K, et al. Mitogen-activated protein (MAP) kinase pathways: regulation and physiological functions. Endocr Rev. 2001;22:153-83.

51. Kyriakis JM, Avruch J. Mammalian mitogen-activated protein kinase signal transduction pathways activated by stress and inflammation. Physiol Rev. 2001;81:807-69.

52. SSlade D. Maneuvers on PCNA Rings during DNA Replication and Repair. Genes (Basel). 2018;9:416

53. Mihaylova MM, Shaw RJ. The AMPK signalling pathway coordinates cell growth, autophagy and metabolism. Nat Cell Biol. 2011;13:1016-23.

54. Jiang X, Tan HY, Teng S, Chan YT, Wang D, Wang N. The Role of AMPActivated Protein Kinase as a Potential Target of Treatment of Hepatocellular Carcinoma. Cancers (Basel). 2019;11:647.

55. Wang S, Sun M, Yu L, Wang Y, Yao Y, Wang D. Niacin inhibits apoptosis and rescues premature ovarian failure. Cell Physiol Biochem. 2018:50:2060-70.

56. Bildik G, Acilan C, Sahin GN, Karahuseyinoglu S, Oktem O. C-Abl is not activated in DNA damage-induced and Tap63-mediated oocyte apoptosis in human ovary. Cell Death Dis. 2018;9:943.

57. Zhang L, Zhang Z, Wang J, LV D, Zhu T, Wang F, et al. Melatonin regulates the activities of ovary and delays the fertility decline in female animals via MT1/AMPK pathway. J Pineal Res. 2019;66:e12550.

\section{Publisher's Note}

Springer Nature remains neutral with regard to jurisdictional claims in published maps and institutional affiliations.
Ready to submit your research? Choose BMC and benefit from:

- fast, convenient online submission

- thorough peer review by experienced researchers in your field

- rapid publication on acceptance

- support for research data, including large and complex data types

- gold Open Access which fosters wider collaboration and increased citations

- maximum visibility for your research: over $100 \mathrm{M}$ website views per year

At $\mathrm{BMC}$, research is always in progress.

Learn more biomedcentral.com/submissions 